\title{
Kinematical coincidence method in transfer reactions
}

\author{
L. Acosta $^{\text {b }}$, F. Amorini ${ }^{\text {b }}$, L. Auditore ${ }^{\text {d }}$, I. Berceanu ${ }^{\text {h }}$, G. Cardella ${ }^{\mathrm{a}, *}$, M.B. Chatterjiee ${ }^{\mathrm{i}}$, E. De Filippo ${ }^{\mathrm{a}}$, \\ L. Francalanza b,c, R. Gianì b,c , L. Grassi a,k, A. Grzeszczuk ${ }^{\mathrm{j}}$, E. La Guidara ${ }^{\mathrm{a}, \mathrm{g}}$, G. Lanzalone ${ }^{\mathrm{b}, \mathrm{e}}$, I. Lombardo ${ }^{\mathrm{b}, \mathrm{f}}$, \\ D. Loria ${ }^{d}$, T. Minniti ${ }^{d}$, E.V. Pagano ${ }^{\text {b,c }}$, M. Papa ${ }^{a}$, S. Pirrone ${ }^{a}$, G. Politi ${ }^{a, c}$, A. Pop $^{\text {h }}$, F. Porto ${ }^{\text {b,c }}$, F. Rizzo $^{\text {b,c }}$, \\ E. Rosato ${ }^{f}$, P. Russotto ${ }^{\text {b,c }}$, S. Santoro ${ }^{d}$, A. Trifirò ${ }^{d}$, M. Trimarchi ${ }^{d}$, G. Verde $^{a}$, M. Vigilante ${ }^{f}$ \\ a INFN-Sezione di Catania, Via S. Sofia, 95123 Catania, Italy \\ ${ }^{\mathrm{b}}$ INFN-Laboratori Nazionali del Sud, Via S. Sofia, Catania, Italy \\ ${ }^{c}$ Dipartimento di Fisica e Astronomia, Università di Catania, Via S. Sofia, Catania, Italy \\ d INFN Gruppo Collegato di Messina and Dipartimento di Fisica, Università di Messina, Italy \\ e Facoltà di Ingegneria e Architettura, Università Kore, Enna, Italy \\ ${ }^{\mathrm{f}}$ Dipartimento di Scienze Fisiche, Università Federico II and INFN Sezione di Napoli, Italy \\ ${ }^{g}$ Centro Siciliano di Fisica Nucleare e Struttura della Materia, Catania, Italy \\ ${ }^{\mathrm{h}}$ Institute for Physics and Nuclear Engineering, Bucharest, Romania \\ i Saha Institute for Nuclear Physics, Kolkata, India \\ ${ }^{\mathrm{j}}$ Institut of Physics, University of Silesia, Katowice, Poland \\ ${ }^{\mathrm{k}}$ Rudjer Boskovic Institute, Zagreb, Croatia
}

\section{A R T I C L E I N F O}

\section{Article history:}

Received 18 December 2012

Received in revised form

14 February 2013

Accepted 13 March 2013

Available online 22 March 2013

Keywords:

Angular distribution

Kinematical coincidence

Radioactive beam

Binary reaction

\begin{abstract}
A B S T R A C T
A new method to extract high resolution angular distributions from kinematical coincidence measurements in binary reactions is presented. Kinematics is used to extract the center of mass angular distribution from the measured energy spectrum of light particles. Results obtained in the case of ${ }^{10} \mathrm{Be}+\mathrm{p} \rightarrow{ }^{9} \mathrm{Be}+\mathrm{d}$ reaction measured with the CHIMERA detector are shown. An angular resolution of few degrees in the center of mass is obtained. The range of applicability of the method is discussed.
\end{abstract}

(c) 2013 Elsevier B.V. All rights reserved.

\section{Introduction}

It is well known how the measurement of angular distributions in elastic scattering and transfer reactions induced by light ions is a very useful method to extract spectroscopic information $[1,2]$. In the last years, these measurements have been carried out in inverse kinematics reactions induced by radioactive beams impinging on light targets by using very performing detection systems, see for instance Refs. [3-9]. One of the most important issues that one has to handle in this type of measurements is the low beam intensity. A possible solution is the one to increase the solid angle coverage, by mounting detectors very close to the target. However, depending on the adopted detector configuration, in this way the angular resolution could be poor, strongly affecting the quality of the experimental results. In this paper, we show that, by using the kinematical coincidence method [10], angular

\footnotetext{
* Corresponding author. Tel.: + 39 0953785321; fax: + 39095337938.

E-mail address: cardella@ct.infn.it (G. Cardella).
}

resolution of the order of $1^{\circ}$ in the center of mass $(\mathrm{CM})$ can be easily achieved without contradiction with the large coverage of the solid angle. This method allows to perform nuclear structure studies also with very powerful $4 \pi$ detector systems, like CHIMERA $[11,12]$, INDRA [13] or the proposed FAZIA [14], that are very efficient to measure kinematical correlations but are generally characterized by poor angular resolution. Also more simple silicon arrays often used as ancillary detectors for gamma ray arrays could be very efficiently used. In this paper we test the power of this method in the study of the angular distributions of the ${ }^{10} \mathrm{Be}+\mathrm{p} \rightarrow{ }^{9} \mathrm{Be}+\mathrm{d}$ reaction at $58 \mathrm{~A} \mathrm{MeV} .{ }^{10} \mathrm{Be}$ beam was produced by using the fragmentation method at INFN Laboratori Nazionali del Sud (INFN-LNS) in Catania [15]. Reaction products were measured with the CHIMERA detector. CHIMERA is a $4 \pi$ detector with a granularity (1192 telescopes) suitable for the study of multifragmentation reactions between heavy ion from 10 to $100 \mathrm{~A} \mathrm{MeV}$. The large segmentation of the apparatus allows to get, at very forward angles, a resolution better than $1^{\circ}$, while, at angles larger than $30^{\circ}$, the angular resolution is $\Delta \theta= \pm 4^{\circ}$. This means, for example, that in the reaction here studied we can get 
approximately $\Delta \theta= \pm 8^{\circ}$ resolution in the CM system for the deuterons angular distribution. The angular resolution is moreover influenced by the emittance of the beam that is induced by the angular straggling of the projectile fragmentation process in the thick production target used. We have a beam spot on the nuclear target of the order of $2 \times 1 \mathrm{~cm}^{2}$ with an angular spread of the beam around $\pm 1^{\circ}$ with a consequent further degradation of the angular resolution. We show in the following how the method used allows also to automatically correct for the angular spread of the beam, obtaining an angular distribution with a resolution substantially influenced only by the statistics of the measurement.

\section{Fragmentation beam characteristics}

The fragmentation beam was produced by using an ${ }^{18} \mathrm{O}^{7+}$ primary beam delivered by the INFN-LNS superconducting cyclotron at $55 \mathrm{~A} \mathrm{MeV}$. The fragmentation reaction was induced on a ${ }^{9} \mathrm{Be}$ target $1.5 \mathrm{~mm}$ thick mounted in the first section of the transport beam line. Following LISE++ simulations [16], the beam line was set to maximize production of ${ }^{11} \mathrm{Be}$ ions $(\mathrm{B} \rho=2.78 \mathrm{Tm})$. The transport of the fragmentation beam was optimized by using the radioactive beam diagnostic system of the INFN-LNS [17]. The beam was identified in particle by particle mode by using a tagging system consisting of two double side silicon strip detectors (DSSSD) and a large surface micro-channel plate (MCP) detector [18]. The first DSSSD detector, hereafter named tagging strip, was $64 \mu \mathrm{m}$ thick with 24 strips on each side and a total surface of $24 \times 24 \mathrm{~mm}^{2}$. It was placed $2 \mathrm{~m}$ before the CHIMERA target and was used to measure the beam energy loss $\Delta E$, and its $X-Y$ position. A second DSSSD detector, named trajectory strip, $72 \mu \mathrm{m}$ thick, $5 \times 5 \mathrm{~cm}^{2}$ surface with 16 strips on each side, was placed about $80 \mathrm{~cm}$ before the CHIMERA target. Being very near $(20 \mathrm{~cm})$ to the entrance hole ( $6 \mathrm{~cm}$ diameter) of the CHIMERA apparatus, the particles produced by reactions in such silicon detector could be a large source of spurious events. Therefore it was used only during beam transport for adjustment purposes and every $12 \mathrm{~h}$ for stability check. In Fig. 1 (a) we plot a sketch (not in scale) of the strip detectors and target ( $50 \mu \mathrm{m}$ thick polyethylene $\mathrm{C}_{2} \mathrm{H}_{4}$ ) in order to define the trajectory measurement. In Fig. 1(b) the calculated beam profile on the target is shown. We observe a small vertical misalignment and an approximate size of $2 \mathrm{~cm}$ along the vertical axis and of $1 \mathrm{~cm}$ along the horizontal one. In Fig. 1(c) and (d) we plot respectively the impinging angle upon the nuclear target $\theta_{\text {beam }}$ as a function of the vertical and horizontal position in the tagging strip. We note a strong correlation between $\theta_{\text {beam }}$ and $x_{\text {strip }}$, with a rather narrow distribution of this angle. This strict correlation is mainly due to the last magnetic dipole of the transport beam line. The MCP detector, $4 \times 6 \mathrm{~cm}^{2}$ wide [18], was placed approximately $13 \mathrm{~m}$ before the tagging strip and was used as start of the time of flight (TOF) measurement of the beam particles (the stop being delivered by the tagging strip ). Notwithstanding the high beam energy we get a reasonably good efficiency of MCP evaporating LiF on the emissive foil [18] (near $90 \%$ for ${ }^{16} \mathrm{C}$ beam). For the ${ }^{10} \mathrm{Be}$ beam, used in the reaction here investigated, an efficiency slightly below $80 \%$ was obtained. In Fig. 2 we show the quality of the identification obtained by plotting the energy loss measured in the tagging strip as a function of the TOF. The isotopic beam identification was obtained from comparison with LISE++ predictions and it was further checked in charge identification and, when possible, in mass by looking to elastic reaction products detected by the forward telescopes of the CHIMERA array.

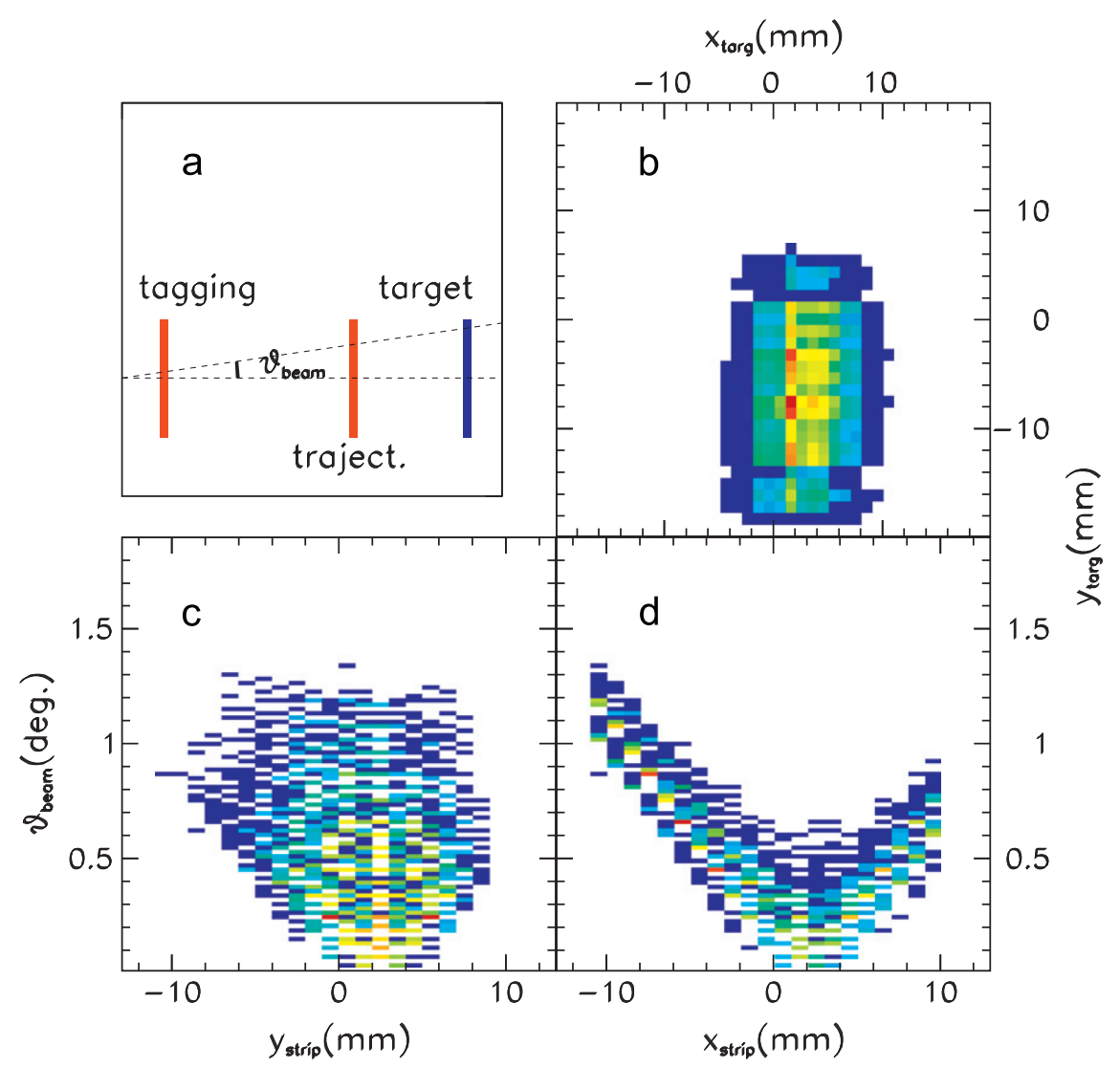

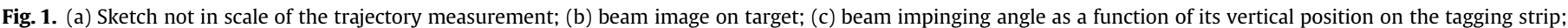
(d) beam impinging angle as a function of its horizontal position in the tagging strip. A clear correlation is observed. 


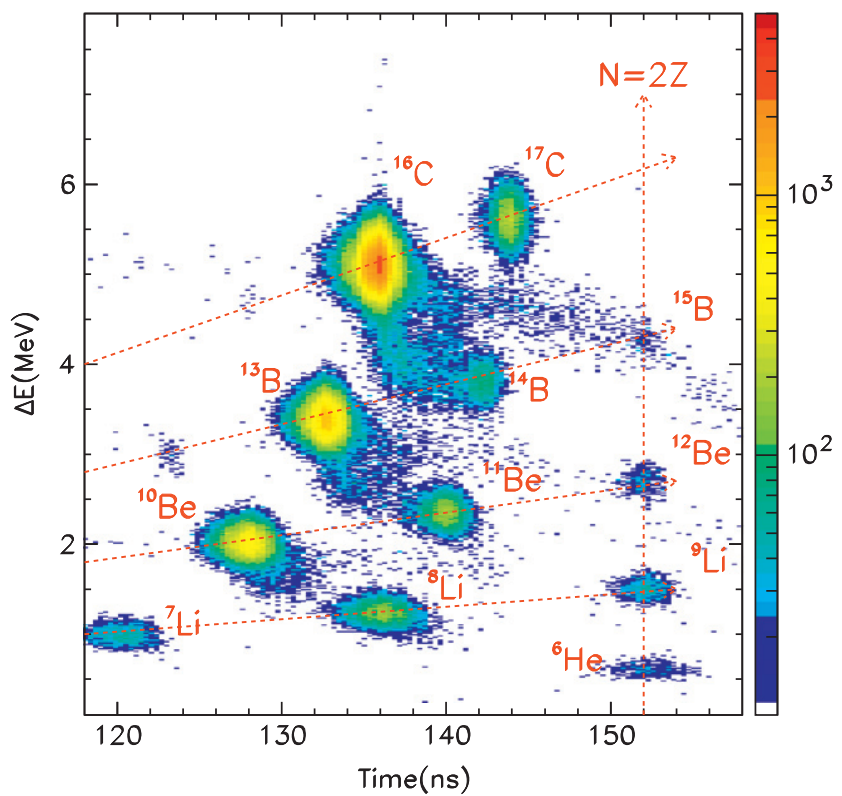

Fig. 2. $\Delta E-T O F$ identification scatter plot of the fragmentation beam used. The arrows show the loci of the different isotopes.

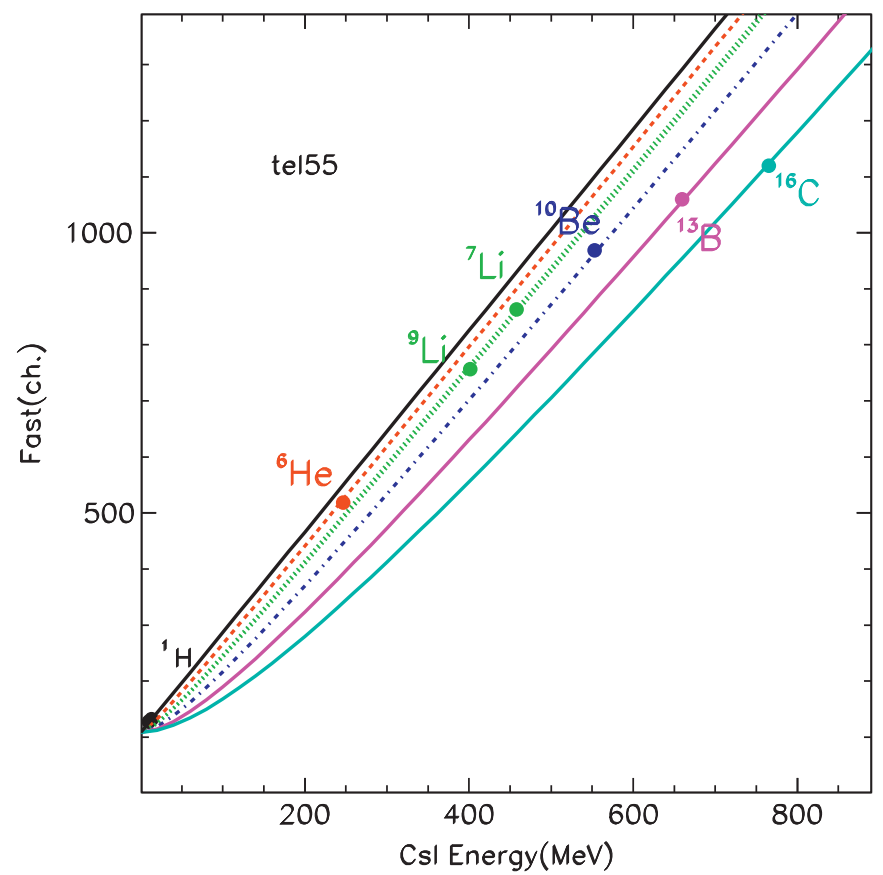

Fig. 3. Calibration points and fit results for one $\operatorname{CsI}(\mathrm{Tl})$ detector of the CHIMERA forward rings.

\section{Calibration procedures}

The individual detection cell of the CHIMERA detector is a telescope constituted by a first stage silicon detector $(300 \mu \mathrm{m}$ thick). The second stage is a $\mathrm{CsI}(\mathrm{Tl})$ scintillator with photodiode readout. Different techniques are used for particle identification allowing for mass and/or charge identification in a large angular and energy range [19-23]. The energy of the impinging particle can be obtained by summing the energy deposited in both detector stages of the telescope. The silicon stage was calibrated in energy by using peaks from low energy light beams elastically scattered by thin Au targets. The situation is more complex for CsI (Tl) detectors due to quenching and non-linearity effects at low kinetic energy in the energy response function. A first order energy calibration of $\mathrm{CsI}(\mathrm{Tl})$ in the forward rings was obtained using the elastic peaks on carbon targets measured with fragmentation beams. Also elastic peaks generated by the scattering of low energy proton beams on carbon and gold targets were used. In Fig. 3 such calibration points are plotted as the $\mathrm{CsI}(\mathrm{Tl})$ total light collected for one detector (Fast variable in channels) [20,21] against the energy deposited in the detector by the particles. In order to take into account the charge and mass dependence of the energy-light response function of detectors we have used the formula suggested by Horn [24]

$$
L=a_{1} E-a_{2} A Z^{2} \ln \left[\left(E+a_{2} A Z^{2}\right) /\left(a_{2} A Z^{2}\right)\right]+a_{0}
$$

where $A, Z$ and $E$ are respectively the mass, charge and energy of the detected fragment, $L$ is the collected light signal, $a_{0}$ is a parameter depending on pedestal, $a_{1}$ is connected to the electronic gain of the channel and includes also the scintillation efficiency of the detector, $a_{2}$ is related to the Birks quenching factor [25]. This formula is based on the assumption that the quenching of $\mathrm{CsI}(\mathrm{Tl})$ light output depends on the specific energy loss of the particle $\mathrm{d} E /$ $\mathrm{d} x$ (Birks prescription). As can be observed in Fig. 3, the fitting parameters allow the reproduction of the general behavior of the experimental response function. After this first order calibration, only second order corrections (few percent) were included, when necessary, for each charge and detector, to better reproduce the energy of elastic and inelastic peaks observed. These corrections take into account small differences in the crystal doping and wrapping, photodiode coupling, and electronics response.

At more backward angles, where practically only light particles were detected, low energy proton elastically scattered on various targets were used for energy calibration. The parameters extracted from the fit of the most forward telescopes and previous data with deuteron beams confirm us that this calibration can be extended, under suitable approximations, to all hydrogen isotopes [26,27]. The energy resolution measured with proton elastic peaks is of the order of $2 \%$.

At very forward angles the standard CHIMERA charge preamplifiers have a conversion sensitivity of $2 \mathrm{mV} / \mathrm{MeV}$, in order to avoid saturation effects due to the expected large dynamical range. Evidently, for the lightest particles this fact does not allow for a clear isotopic identification by using the $\Delta E-E$ method, as instead obtained at larger angles [19]. Therefore, apart from a few very well performing telescopes, in the forward direction we had only charge identification for the heavy reaction partners.

Particles emitted at angles larger than $20^{\circ}$ were identified in charge and mass with $\Delta E-E$ method and for $Z \leq 2$ also with fastslow method, Fig. 4, allowing good separation also at high particle energy where the energy loss in silicon detector is small and $\Delta E-E$ method is consequently less reliable.

\section{Data analysis and results}

As it is seen in Fig. 2a "cocktail" of fragmentation beams are identified in the tagging strip. Thus the first step of the data analysis consists on the selection of the beam under study $\left({ }^{10} \mathrm{Be}\right.$ in this case) with the use of appropriate cuts. Events were further selected, searching for d-Be coincidences with Beryllium ions (charge identification) detected in the most forward rings and deuteron ions ( isotopic identification) in the angular range which was allowed by kinematics. Only events with charged particles multiplicity equal to two were analyzed, strongly reducing contamination due to carbon in the polyethylene target, and to reactions in the tagging detector. Other constraints were taken into account by using conservation laws. Firstly, due to momentum conservation, the relative azimuthal angle $\Delta \phi$ between the 


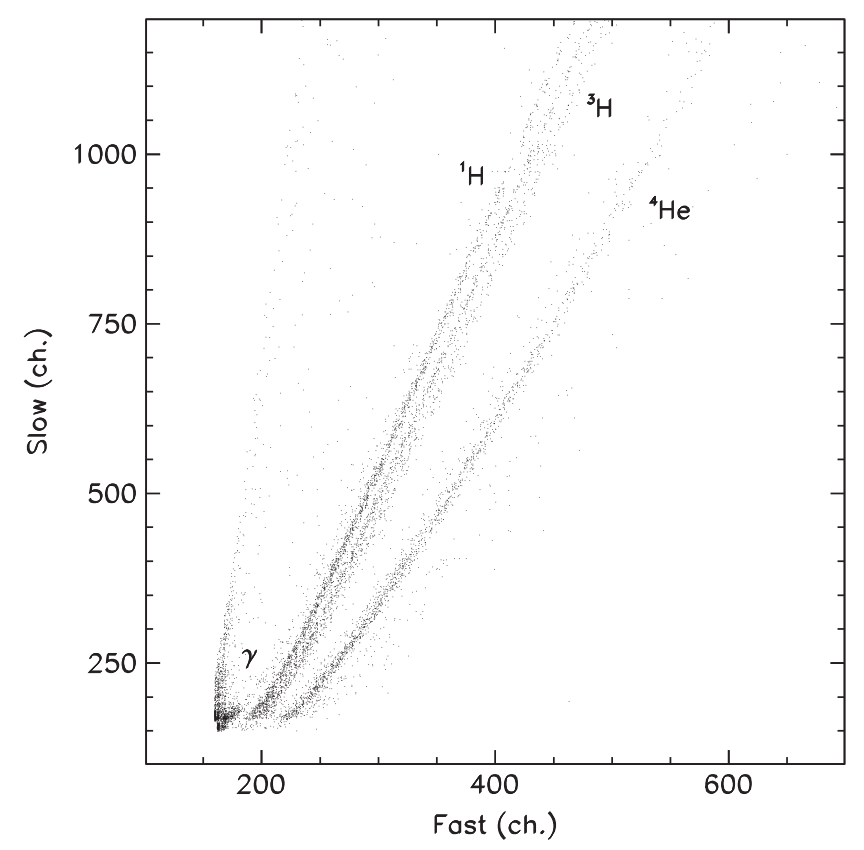

Fig. 4. Fast-slow scatter plot of a telescope at $34^{\circ} \cdot \gamma$-Rays, proton, deuteron tritons and $\alpha$-particles are identified.

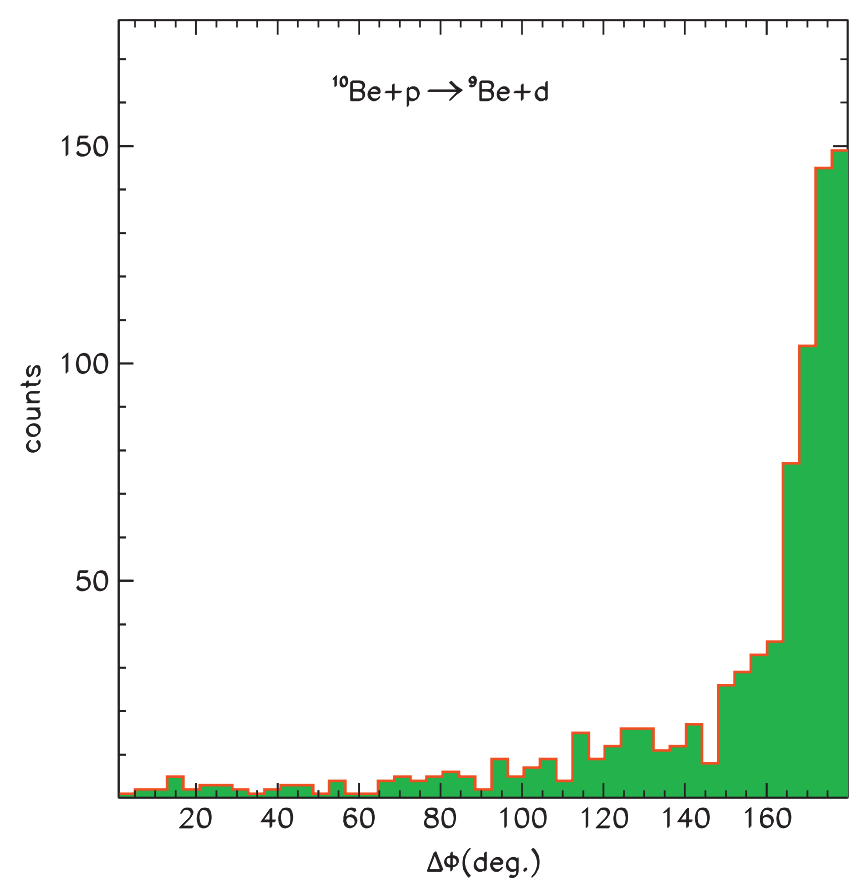

Fig. 5. Relative angle $\Delta \phi$ between the telescopes selected in coincidence in the reaction ${ }^{10} \mathrm{Be}+\mathrm{p} \rightarrow{ }^{9} \mathrm{Be}+\mathrm{d}$. The peak at $180^{\circ}$ is due to kinematical coincidences.

two fragments must be $180^{\circ}$. In Fig. 5, we plot this angle as measured for the investigated reaction, ${ }^{10} \mathrm{Be}+\mathrm{p} \rightarrow{ }^{9} \mathrm{Be}+\mathrm{d}$. We can recognize the binary events concentrated in the peak around $180^{\circ}$. The width at half maximum of this peak is about $16^{\circ}$, mainly due to the $\phi$ opening angle of detectors.

A further selection, based on energy conservation law, requires that the sum of kinetic energies of the two detected particles is equal to the beam energy plus the $Q$-value $(Q=-4.58 \mathrm{MeV}$ ) (see Fig. 6). Notice in this plot a peak close to the value of $580 \mathrm{MeV}$, that roughly corresponds to the total available energy. Due to the relatively poor $\mathrm{CsI}(\mathrm{Tl})$ energy resolution for heavy fragments, it is

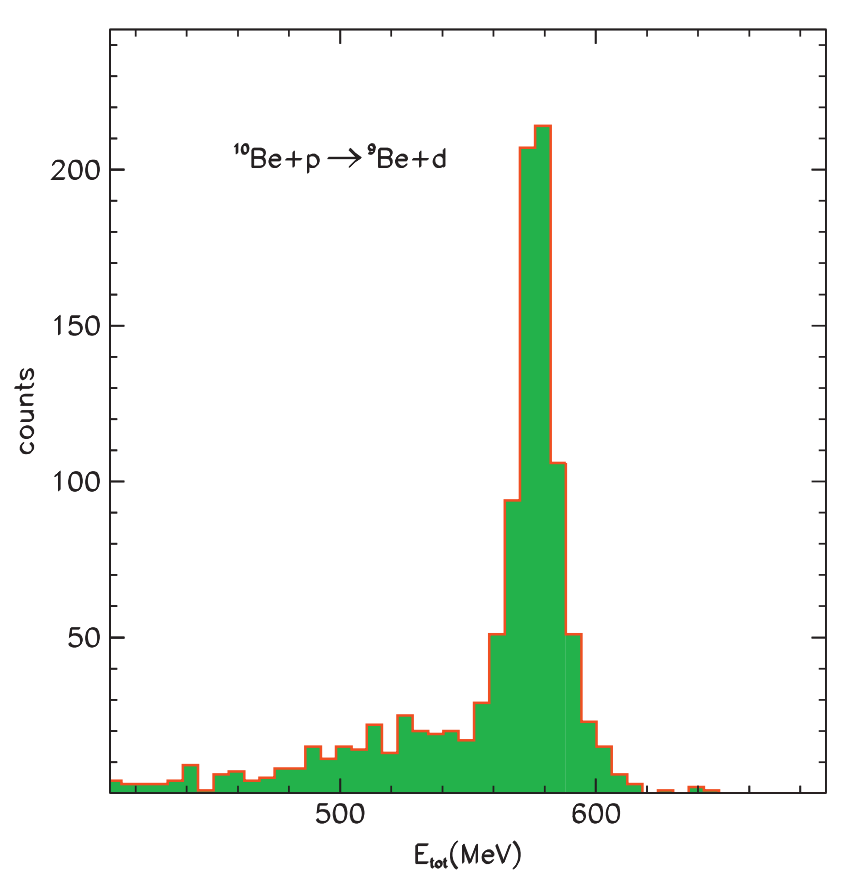

Fig. 6. Total kinetic energy detected in the reaction ${ }^{10} \mathrm{Be}+\mathrm{p} \rightarrow{ }^{9} \mathrm{Be}+\mathrm{d}$.

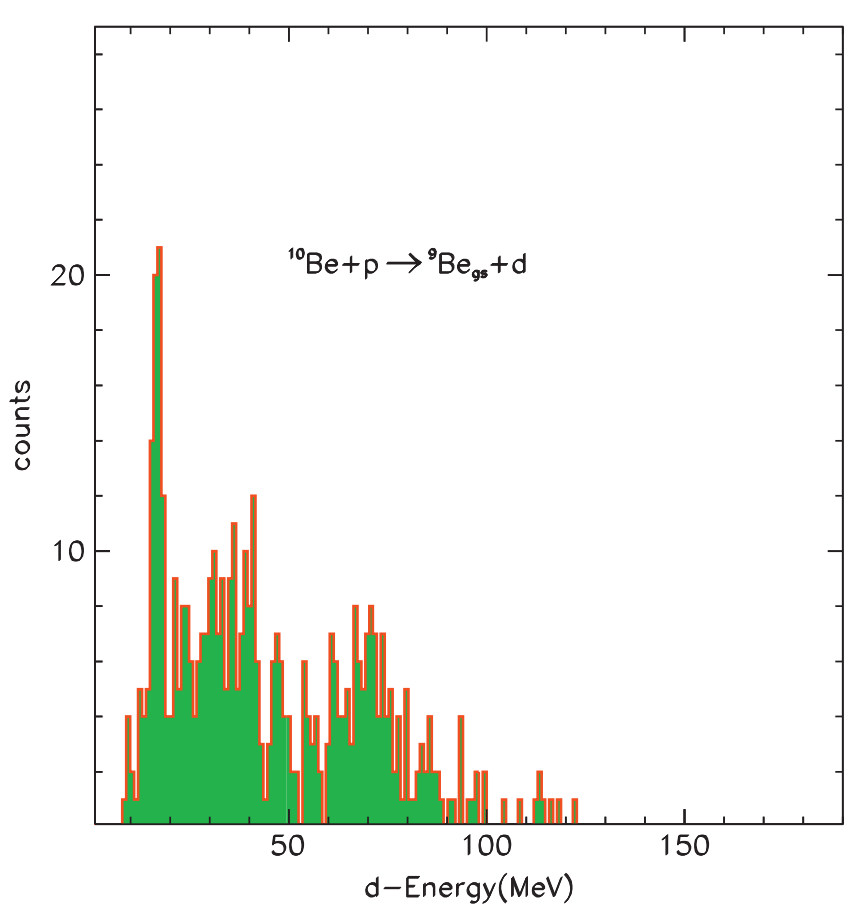

Fig. 7. Deuteron energy spectrum from the reaction ${ }^{10} \mathrm{Be}+\mathrm{p} \rightarrow{ }^{9} \mathrm{Be} g s+\mathrm{d}$.

quite difficult to discriminate the decay path towards to the ground state of a specific nucleus with respect to excited states by just looking only at the peak in the total energy spectrum. However, in the simple case here investigated, the neutron separation energy for ${ }^{9} \mathrm{Be}$ is only $1.665 \mathrm{MeV}$, and even the first excited level $(1.684 \mathrm{MeV})$ is unbound, decaying to the $n+2 \alpha$ channel. Therefore, by requiring a beryllium in the final channel we rejected excited levels in a natural way. We conclude that we are observing only the GS level.

In Fig. 7 we finally plot the deuteron energy spectrum as it was measured for this channel. The low energy part of the spectrum is 


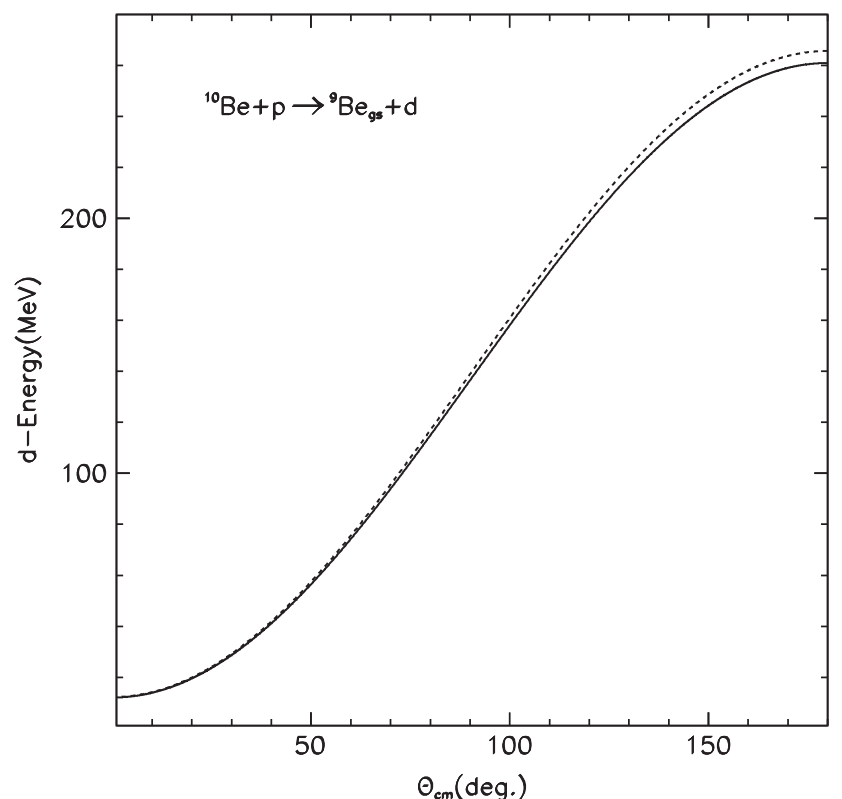

Fig. 8. Kinematical correlation between the deuteron energy and the $\theta_{\mathrm{cm}}$ in the reaction ${ }^{10} \mathrm{Be}+\mathrm{p} \rightarrow{ }^{9} \mathrm{Be}_{\mathrm{gs}}+\mathrm{d} 58 \mathrm{~A} \mathrm{MeV}$ (full line). Dashed line is computed for a beam energy of $59 \mathrm{~A} \mathrm{MeV}$.

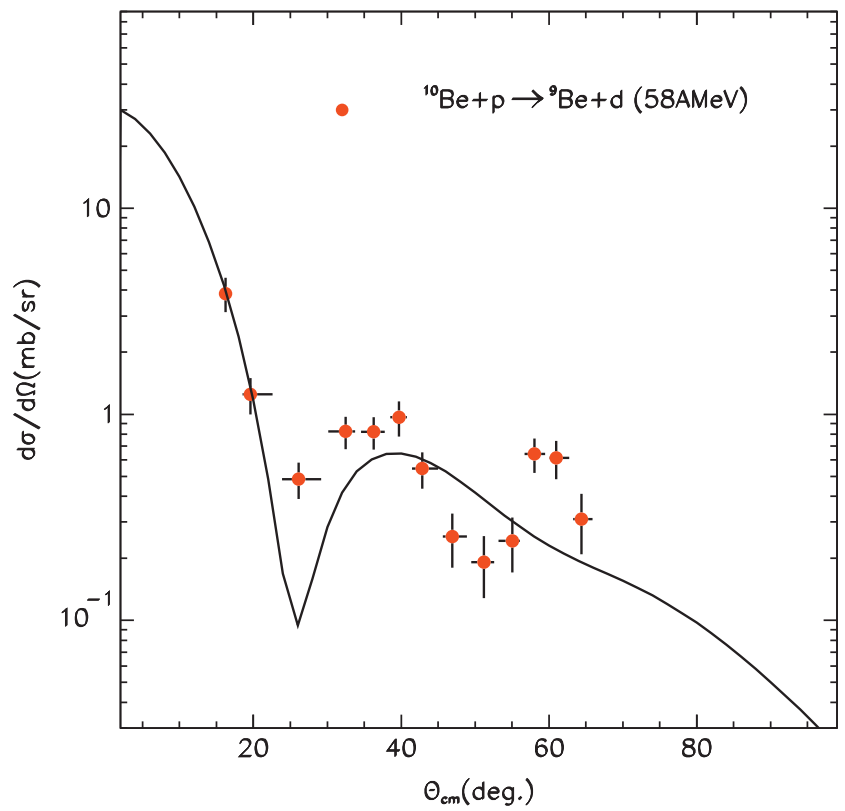

Fig. 9. Angular distribution converted from the deuteron energy spectrum of Fig. 7 (full dots). The line is a standard DWBA calculation following Ref. [32].

affected by efficiency problems better described below. It is interesting to observe the presence of two relative minima near 20 and $50 \mathrm{MeV}$. Since in this case the final channel is well defined, we can easily convert this energy spectrum in the $\mathrm{CM}$ angular distribution by using the kinematic relation, shown in Fig. 8. By using such relation we can convert the $\Delta E$ energy interval of each channel in Fig. 7 in angular interval $\Delta \theta_{\mathrm{i}}$ directly in the CM system. With this method we can therefore deduce the number of particles detected in the $\Delta \theta_{\mathrm{i}}$ angular range. Dividing this number for the solid angle subtended by the arc of sphere $\Delta \theta_{\mathrm{i}}$ and taking into account the number of the beam particles $\left(7.3 \times 10^{8}\right)$ and the areal density of the target nuclei, we can get the absolute cross section.

We underline that using the deuteron energy to determine the center of mass angle, we automatically correct for the spread of the beam impinging position and angle described in paragraph 2, Fig. 1.

Obviously we have to include also efficiency corrections. We have to note that in this experiment some CHIMERA rings between $7^{\circ}$ and $20^{\circ}$ in the laboratory frame were missing because in use to another experiment [28]. Thus a relevant correction has to be introduced. According to the simulations, $100 \%$ efficiency was maintained only from $\theta_{\mathrm{cm}} \approx 20^{\circ}$ to $\theta_{\mathrm{cm}} \approx 60^{\circ}$. However due to the fragmentation beam angular spread, already included in the simulation, the efficiency decreasing with the angle is rather smooth and we can observe coincidences in the CM angular range from approximately $15^{\circ}$ to $70^{\circ}$. In Fig. 9 we plot the efficiency corrected angular distribution (full dots). Only statistical error is plotted. The minima evidenced in the deuteron energy spectrum are clearly converted into minima in the angular distribution. The value of the size of the angular bins of each point was mainly governed by the need to get a reasonable statistical error. In the analysis of the errors on the cross section evaluation we can completely neglect the impinging beam intensity nearly perfectly measured with the tagging system. The main uncertainty arises from the chosen integration range of $\Delta \phi\left(160^{\circ}<\Delta \phi<180^{\circ}\right)$. Due to the beam spread and to the angular straggling in the relatively thick target, true coincidences could be lost. This error can be evaluated to be up to $15 \%$ by integrating the neighbors of the main peak. Carbon contamination is instead negligible because it is largely suppressed by the total detected charge measurement. Also random coincidences are few, due to the low beam rate and the rather narrow timing acceptance window obtained with our TDCs. Only very forward detectors show some random coincidences that are practically all suppressed by the efficiency correction stage of the analysis requesting a coherence between the angular range of the detectors, the kinematics and the angular spread of the beam. Another 5\% error can be evaluated due to the inhomogeneity of the target thickness.

To our knowledge, there are no previous data on this angular distribution available in the literature. Only data on the inverse reaction are available [29], at different center of mass energies, showing similar oscillating behavior with small changes in the position of minima. It is also interesting to compare our data with the angular distribution measured by Auton [30], recently reanalyzed in [31], for the reaction $\mathrm{d}+{ }^{10} \mathrm{Be} \rightarrow \mathrm{t}+{ }^{9} \mathrm{Be}_{\mathrm{gs}}$ at $15 \mathrm{MeV}$ deuteron beam energy. The one-neutron transfer mechanisms involves in fact the same orbitals but the presence of another neutron in the deuteron target and its pairing interaction with the transferred neutron could produce some difference. However even in this case similar minima are observed. A very preliminary DWUCK5 calculation performed with standard parameters using the tools of NRV project [32] is plotted as continuous line. The cross section reproduction at forward angles is rather good, also the first minimum at $26^{\circ}$ and the maximum around $40^{\circ}$ are relatively well reproduced. The disagreement at larger angles needs a much more detailed analysis as the one performed in Ref. [31] that cannot be fully discussed in this paper and will be the object of future work.

\section{Perspectives and conclusions}

The method here used rely on the energy of the light detected particle to extract the angular distribution of the reaction, once the decay channel is determined. Therefore it could be also used in $\gamma-$ particle coincidence measurements if the $\gamma$-rays allow to determine the excited level investigated. In general it can be useful in all cases where a strong dependence of the particle energy against detection angle is present as in inverse kinematics reactions. 


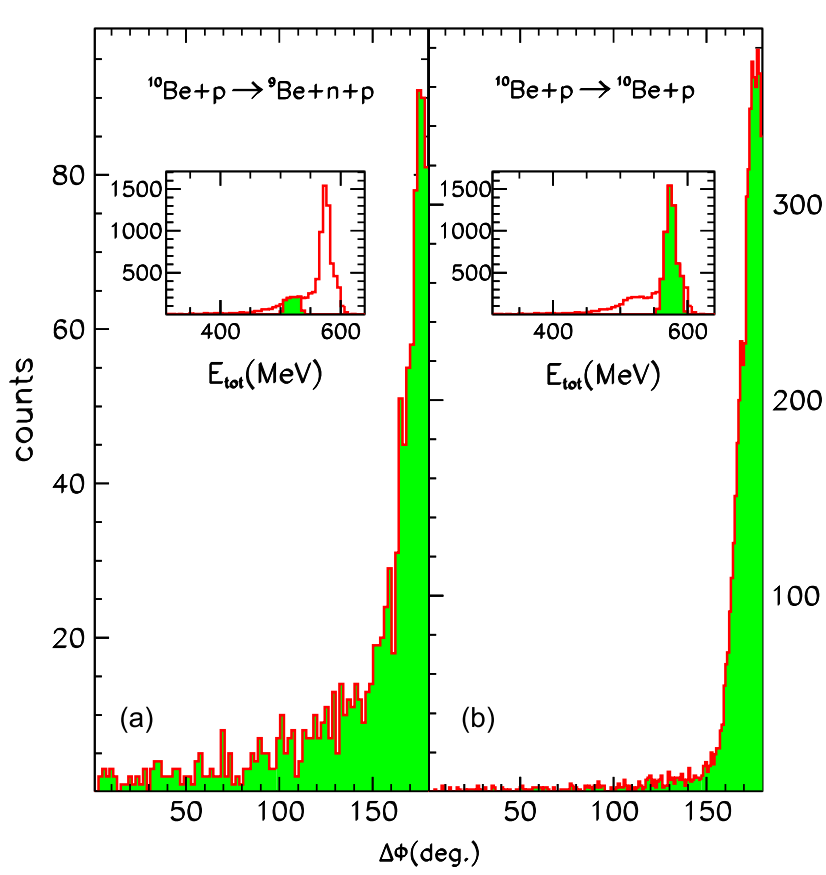

Fig. 10. $\Delta \phi$ distribution of coincidence $\mathrm{Be}+\mathrm{p}$ events in ${ }^{10} \mathrm{Be}+\mathrm{p}$ reactions. (a) Events selected with total energy windows (shown in the insets) corresponding to excited ${ }^{10} \mathrm{Be}$ decaying to ${ }^{9} \mathrm{Be}+\mathrm{n}$. (b) Events selected in the "elastic" channel. The larger width of the $\Delta \phi$ distribution shown in panel (a) is mainly due to the neutron decay.

In the experiment here analyzed a limitation was the poor resolution of the energy measurement for the heavy reaction partner. This is due to the relatively poor energy resolution of $\mathrm{CsI}(\mathrm{Tl})$ detectors. This point can be improved decreasing the beam energy and using triple telescopes, with two stages silicon double sided strip detectors, with 300 and $1500 \mu \mathrm{m}$ thickness respectively, as the new FARCOS telescopes we are building [33]. Using such kind of telescopes at lower energy (20 A MeV) the heavy ion can be stopped in the second stage, so the energy will be measured with the usual excellent resolution of silicon detectors. Also the mass identification and $\phi$ resolution guaranteed by such highly segmented telescopes would greatly improve data quality.

The kinematical coincidence is very narrow in the case of binary reactions, it is however not completely destroyed also in case of secondary particle emission. In Fig. 10(a) and (b) we compare the $\Delta \phi$ distributions in Be-proton coincidence events, for different windows of total energy (shown as full histograms in the insets). The $\Delta \phi$ distribution relative to the "elastic" channel (Fig. 10b) is rather narrow with very small background and can be well fitted with a Gaussian distribution with sigma around $10^{\circ}$. The $\Delta \phi$ distribution (Fig. 10a) relative to the second bump of the total energy distribution is still a peak even if with a not fully Gaussian shape and a larger width. These events are mainly due to the $1 \mathrm{n}$ decay channel of excited ${ }^{10} \mathrm{Be}$. The total energy is in fact approximately $50 \mathrm{MeV}$ smaller because the emission energy of the neutron is missing (approximately the beam energy). In the CM system the emission velocity of the neutron is however rather small compared to the center of mass one, so the spread of the $\Delta \phi$ peak is not so much larger. Therefore the method can be still used even if with a worsened signal to noise ratio. Obviously it would be nice to have mass identification of the heavy partner, better energy resolution and/or gamma ray tagging in order to reduce the background. A better granularity in $\phi$ could also be useful to determine the average decay energy of the undetected neutron. This could be for instance a quite interesting case for the study of resonances at the boundary of the drip lines where unstable nuclear resonances as ${ }^{10} \mathrm{Li}$ can be populated.

In summary, we have shown that detailed angular distributions can be extracted in binary reactions induced by exotic nuclei impinging on light targets by using the kinematical coincidence method. The deuteron energy resolution of our data is of the order of $1 \mathrm{MeV}$ as evaluated by elastic scattering of protons. The approximation on the deuteron energy calibration is of the same order of magnitude. This energy resolution in the light particle energy spectrum is enough to induce a $\mathrm{CM}$ angular resolution better than $1^{\circ}$ due to the approximately linear correlation over a large angular range between these two quantities. This method has the great advantage to automatically correct for the angular spread of the impinging fragmentation radioactive beam. Note also that the relatively large energy spread of the fragmentation beam $(\Delta P / P=1 \%)$ produces in our case a very small effect, seen only at very backward $C M$ angles, see Fig. 8 dashed line. This effect was neglected for the purpose of this paper. This presented method does not need a very high resolution in the measurement of the energy of the heavy fragment, around Fermi energy, if nuclei with only unbound excited levels are investigated as in the case of the ${ }^{9} \mathrm{Be}$. The method can be however extended also to the case of bound and unbound excited levels especially if coincidence gamma ray measurements are performed allowing for discrimination of the different contribution of the decay process.

Thanks are due to Dr. A. Pagano for various discussions and his help and encouraging suggestions since the beginning of this work.

\section{References}

[1] I. Tanihata, H. Savajols, R. Kanungod, Progress in Particle and Nuclear Physics 68 (2013) 215, and references therein.

[2] W. Mittig, P. Roussel-Chomaz, Nuclear Physics A 693 (2001) 495, and references therein.

[3] Y. Blumenfeld, et al., Nuclear Instruments and Methods A 421 (1999) 471.

[4] E.C. Pollacco, et al., European Physical Journal A 25 (2005) 287.

[5] M.S. Wallace, et al., Nuclear Instruments and Methods A 583 (2007) 302.

[6] A.H. Wuosmaa, et al., Nuclear Instruments and Methods A 580 (2007) 1290; B.B. Back, et al., Physical Review Letters 104 (2010) 132501.

[7] A. Di Pietro, et al., Physical Review Letters 105 (2010) 022701.

[8] N. Patronis, et al., Physical Review C 85 (2012) 024609.

[9] L. Acosta, et al., Physical Review C 84 (2011) 044604.

[10] G. Casini, P.R. Maurenzig, A. Olmi, A.A. Stefanini, Nuclear Instruments and Methods A 277 (1989) 445.

[11] A. Pagano, et al., Nuclear Physics A 734 (2004) 504.

[12] A. Pagano, Nuclear Physics News 22 (1) (2012) 25.

[13] J. Pouthas, et al., Nuclear Instruments and Methods A 357 (1995) 418.

[14] S. Carboni, et al., Nuclear Instruments and Methods A 664 (2012) 251

[15] G. Raciti, et al., Nuclear Instruments and Methods B 266 (2008) 4632.

[16] O.B. Tarasov, D. Bazin, Nuclear Instruments and Methods B 266 (2008) 4657.

[17] G. Cosentino et al., LNS report (2009).

[18] I. Lombardo, et al., Nuclear Physics B (Proceedings Supplements) 215 (2011) 272.

[19] N. Le Neindre, et al., Nuclear Instruments and Methods A 490 (2002) 251.

[20] M. Alderighi, et al., Nuclear Instruments and Methods A 489 (2002) 257.

[21] F. Amorini, et al., IEEE Transactions on Nuclear Science 59 (2012) 1772.

[22] S. Aiello, et al., Nuclear Instruments and Methods A 385 (1997) 306.

[23] R. Bassini, et al., IEEE Transactions on Nuclear Science 53 (2006) 507.

[24] D. Horn, et al., Nuclear Instruments and Methods A 320 (1992) 273.

[25] J.B. Birks, The Theory and Practice of Scintillation Counting, Pergamon, New York, 1964, p. 465.

[26] A. Wagner, et al., Nuclear Instruments and Methods A 456 (2001) 290.

[27] I. Lombardo, Degree Thesis, unpublished.

[28] P. Russotto, et al., Proceedings of 11th International Conference on Nucleus Nucleus Collisions, IOP Conference Proceedings 420 (2013) 012092.

[29] R.J. Slobodrian, Physical Review C 126 (1962) 1059; J.P. Schiezer, G.C. Morrison, R.H. Siemsseb, B. Zeidman, Physical Review C 164 (1967) 1274.

[30] D.L. Auton, Nuclear Physics A 157 (1970) 305

[31] N. Keeley, K.W. Kemper, K. Rusek, Physical Review C 86 (2012) 014619.

[32] V. Zagrebaev, et al., DWBA Code of $\langle$ http://nrv.jinr.ru/nrv/ $\rangle$.

[33] L. Acosta, et al., EPJ Web of Conferences, 310,035 (2012). 